\title{
Reactions of $\mathrm{MoOCl}_{4}$ and $\mathrm{MoO}_{2} \mathrm{Cl}_{2}$ with Heterocyclic Thioamides
}

\author{
DEEPIKA RANI ${ }^{1}$, GURSHARAN SINGH ${ }^{2 *}$ and SEEMA SHARMA ${ }^{2}$
}

\author{
${ }^{1}$ Research Scholar registered with Punjab Technical University, Kapurthala, India. \\ 2Department of Applied Chemistry, Giani Zail Singh Campus College of Engineering \\ \& Technology, Dabwali Road, MRSPTU Bathinda-151001, India. \\ *Corresponding author E-mail: gursharans82 @ gmail.com
}

http://dx.doi.org/10.13005/ojc/370228

(Received: March 17, 2021; Accepted: April 18, 2021)

\begin{abstract}
$\mathrm{MoOCl} / \mathrm{MoO}_{2} \mathrm{Cl}_{2}$ were reacted with 2-mercaptopyridine (IUPAC: pyridine-2-thiol)/4phenylimidazole-2-thiol (IUPAC: 4-phenyl-1,3-dihydroimidazole-2-thione)/6-mercaptopurine (IUPAC: 1,7-dihydro-purine-6-thione) in acetonitrile medium using equal/twice molar concentrations at normal temperature. The reactions yielded products: $\mathrm{MoOCl}\left(\mathrm{C}_{5} \mathrm{H}_{4} \mathrm{NS}-\mathrm{SNH}_{4} \mathrm{C}_{5}\right) \cdot 2 \mathrm{HCl},[1] ; \mathrm{Mo}_{3} \mathrm{O}_{3} \mathrm{Cl}_{12}\left(\mathrm{C}_{5} \mathrm{H}_{4} \mathrm{NS}-\right.$ $\left.\mathrm{SNH}_{4} \mathrm{C}_{5}\right)\left(\mathrm{CH}_{3} \mathrm{CN}\right)_{2}$, [2]; $\mathrm{Mo}_{2} \mathrm{OCl}_{6}\left(\mathrm{C}_{9} \mathrm{H}_{7} \mathrm{~N}_{2} \mathrm{~S}\right)_{4}$, [3] and $\mathrm{Mo}_{2} \mathrm{O}_{2} \mathrm{Cl}_{8}\left(\mathrm{C}_{5} \mathrm{H}_{4} \mathrm{~N}_{4} \mathrm{~S}\right)\left(\mathrm{CH}_{3} \mathrm{CN}\right)$, [4]. The various techniques used for characterization of compounds are: Fourier transform infrared, protron nuclear magnetic resonance, ${ }^{13} \mathrm{C}$ nuclear magnetic resonance, liquid/gas chromatography, mass proton spectrometry and C, H, N, S, Mo, Cl analysis. The products are prone to oxidation/hydrolysis by air/ moisture, so all procedures were executed in vacuum line using dry nitrogen atmosphere. Elemental analysis and fragments recorded in mass spectrometry are in tune with the formulae proposed.
\end{abstract}

\begin{abstract}
Keywords: 2-mercaptopyridine (IUPAC: pyridine-2-thiol), 4-phenylimidazole-2-thiol (IUPAC: 4-phenyl-1,3-dihydroimidazole-2-thione), 6-mercaptopurine (IUPAC: 1,7-dihydro-purine-6thione), $\mathrm{MoOCl}_{4}, \mathrm{MoO}_{2} \mathrm{Cl}_{2}, \mathrm{FTIR},{ }^{1} \mathrm{H} \mathrm{NMR},{ }^{13} \mathrm{C} \mathrm{NMR}$, DMSO-d 6 , liquid/gas chromatography-mass spectrometry, Acetonitrile medium.
\end{abstract}

\section{INTRODUCTION}

\section{6-Mercaptopurine (IUPAC: 1,7-dihydro-purine- 6-thione)}

Pyrimidine ring and imidazole ring are fused in purine. 6-mercaptopurine $(\mathrm{mpH})$ has delocalized $\pi$-electrons. Nucleophiles can attack at positions 2,6 and 8. Electrophiles can attack at positions 3 and 7.

Purine ring system represents skeleton for nucleic acid bases guanine and adenine. 6-Mercaptopurine $\mathrm{e}^{1-2}$ is used as chemotherapy drugs to treat various types of cancers. This drug is also used to cure autoimmune diseases. 6-Mercaptopurine is marketed as purinethol. Purine is antagonists drug because it stops the growth of cancer cells.

6-Mercaptopurine reacts with many transition metals ${ }^{3-4}$. Some transition metal complexes of the ligand are more potent against cancer than the ligand itself5-8. 6-Mercaptopurine coordinates ${ }^{5,7,9}$ via sulphur and nitrogen atoms with divalent transition metals. 6-Mercaptopurine $(\mathrm{mpH})$ reacts with various transition metal ions in different coordination ways: $\mathrm{mpH}^{+}, \mathrm{mp}^{-}, \mathrm{mp}^{2-}$ and intramolecular proton transfer.

This is an Open Access article licensed under a Creative Commons license: Attribution 4.0 International (CC- BY). Published by Oriental Scientific Publishing Company @ 2018

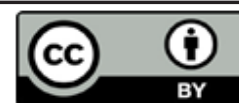


There is thione-thiol tautomerism. In gaseous state, thiol form is favoured, whereas in solution form thione form is favoured ${ }^{10}$. There is also prototropic tautomerism ${ }^{10}$ in 6-mercaptopurine between 7 and 9 positions.<smiles>S=c1[nH]cnc2nc[nH]c12</smiles>

6-Mercaptopurine

4-Phenylimidazole-2-thiol (IUPAC: 4-phenyl-1, 3-dihydroimidazole-2-thione)

Thioamides have higher reactivity ${ }^{11}$ than ordinary amides, because (a) thioamides have lesser $\mathrm{C}=\mathrm{S}$ double bond character than $\mathrm{C}=\mathrm{O}$ due to larger size of $S$ atom than that of $C$ atom and bond dissociation energy of $\mathrm{C}=\mathrm{O}$ bond is $50 \mathrm{k} . c a l . / \mathrm{mole}$ higher than that of $\mathrm{C}=\mathrm{S}$ bond, (b) greater participation of the sulphur to lower occupied molecular orbitals of the thioamide group, (c) there is more electron density on nitrogen atom in thioamides, so protons on the nitrogen atom is are more labile, (d) higher lability of the protons on carbons next to the thioamide group. The sulphur atom of thioamide group accepts nucleophiles and electrophiles.

Heterocyclic thioamides are used in antithyroidal drugs ${ }^{12}$. Heterocyclic imidazothiazole are biologically active compounds ${ }^{13}$. They are able to inhibit many receptors and enzymes ${ }^{14-17}$. They have applications as diuretic ${ }^{18}$, antihelmintic ${ }^{19}$, fungicidal ${ }^{20}$, antidiabetic ${ }^{21}$, antitumor ${ }^{22-27}$ and antimicrobial ${ }^{28-29}$ drugs. Thioligand metal complexes have biochemical applications ${ }^{30-32}$. They are also used as analytical reagents or metal corrosion inhibitors ${ }^{12}$.<smiles>Sc1nc(-c2ccccc2)c[nH]1</smiles>

\section{2-Mercaptopyridine (IUPAC: pyridine-2-thiol)}

2-Mercaptopyridine and $1 \mathrm{H}$-pyridine2-thione are tautomers. We can represent 2-mercaptopyridine as pySH. There are two probable coordination sites ${ }^{11}$ pySH and pyS-(pyridine-2thiolate ion), but pySH is more prevalent with transition metals. pyS- can also act as bridging ligand. pyS-(pyridine-2-thiolate ion) further tautomerise ${ }^{11}$ to pyridine-2-thioamidate ion with negative charge on nitrogen atom.

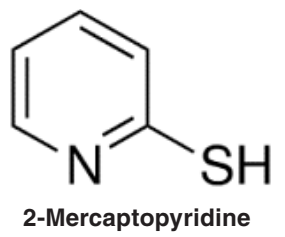

Aim of investigation

Many ligands have been found to react with $\mathrm{MoO}_{2} \mathrm{Cl}_{2}$ and $\mathrm{MoOCl}_{4}$.

Many complexes of alkanediols, amides, aromatic azoles, diaminoalkanes, imides, purine, thioamides and thiols with $\mathrm{MoO}_{2} \mathrm{Cl}_{2}$ have been reported ${ }^{33-40}$ by the author.

Many complexes of alkylpyridines, amides, alkylpyrrolidine, alkylpiperidine, aromatic azoles, diaminoalkanes, imides, mercaptopyridine, mercaptopyridine- $\mathrm{N}$-oxide sodium, purine and 2-thiazoline-2-thiol with $\mathrm{MoOCl}_{4}$ have also been reported ${ }^{33-35,40-42}$ by the author.

Since complexes of these ligands with transition metals have many applications, we have synthesized complexes of 2-mercaptopyridine (IUPAC: pyridine-2-thiol)/4-phenylimidazole-2-thiol (IUPAC: 4-phenyl-1, 3-dihydroimidazole-2-thione)/6mercaptopurine (IUPAC: 1,7-dihydro-purine-6thione) with precursors $\mathrm{MoOCl}_{4}$ and $\mathrm{MoO}_{2} \mathrm{Cl}_{2}$. The various techniques used for characterization of compounds are: Fourier transform infrared, proton nuclear magnetic resonance, ${ }^{13} \mathrm{C}$ nuclear magnetic resonance, liquid/gas chromatography mass spectrometry and $\mathrm{C}, \mathrm{H}, \mathrm{N}, \mathrm{S}, \mathrm{Mo}, \mathrm{Cl}$ analysis. The products are prone to oxidation/hydrolysis by air/ moisture, so all procedures were executed in vacuum line using dry nitrogen atmosphere.

\section{MATERIALS AND METHODS}

Synthesis of $\mathrm{MoOCl}_{4}$ was carried out from thionyl chloride and molybdenum trioxide at reflux temperature. Unreacted thionyl chloride was evacuated into liquid nitrogen cooled traps. Dark green crystals of $\mathrm{MoOCl}_{4}$ isolated on evacuation were dissolved in dry dichloromethane. Dark red solution was obtained. It was filtered to eliminate 
any unreacted/undissolved $\mathrm{MoO}_{3}$. Red solution was evaporated to get dark green crystals of $\mathrm{MoOCl}_{4}$.

2-Mercaptopyridine, 4-phenylimidazole-2thiol, 6-mercaptopurine monohydrate and $\mathrm{MoO}_{2} \mathrm{Cl}_{2}$ were procured from Sigma-Aldrich and used as such.

The products are prone to oxidation/ hydrolysis by air/moisture, so all procedures were executed in vacuum line using dry nitrogen atmosphere. Ligand solution in dry dichloromethane acetonitrile was added dropwise to $\mathrm{MoOCl}_{4} / \mathrm{MoO}_{2} \mathrm{Cl}_{2}$ with continuous stirring from pressure stabilised dropping funnel. The reactants were agitated for 6-7 hours. The products were filtered through G-4 sintered glass crucible filtration unit and isolated.

Oxinate ${ }^{43}$ gravimetric method was used in the lab for molybdenum estimation. Silver chloride ${ }^{43}$ gravimetric method was used in the lab for chlorine estimation. Remaining elements were analysed with Thermo Finnigan Elemental Analyser. Infrared spectra were recorded with Perkin-Elmer 400 FTIR Spectrometer. Proton nuclear magnetic resonance, ${ }^{13} \mathrm{C}$ nuclear magnetic resonance spectra in DMSO- $\mathrm{d}_{6}$ were taken with Multinuclear Brucker Avance-II 400 NMR spectrometer. Liquid/Gas Chromatography-
Mass spectra were recorded in the range $0-1100 \mathrm{~m} / \mathrm{z}$. Above instruments were used at P. U. Chandigarh.

\section{Reactions}

It is probable that mechanism of these reactions involve oxidation/reduction, disproportionation/rearrangement, isomerization steps. The product source residue $(\mathrm{R})$ or filtrate $(\mathrm{F})$ is shown below the products.

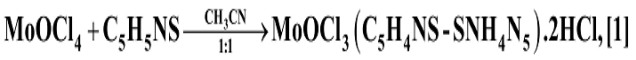

$$
\begin{aligned}
& \text { 2-Mercaptopyridine Light bluegreen }(R) \\
& \mathrm{MoOCl}_{4}+\mathrm{C}_{5} \mathrm{H}_{5} \mathrm{NS} \underset{\mathrm{l}: 1}{\stackrel{\mathrm{CH}}{\mathrm{CN}}} \longrightarrow \mathrm{MO}_{3} \mathrm{O}_{3} \mathrm{Cl}_{12}\left(\mathrm{C}_{5} \mathrm{H}_{4} \mathrm{NS}-\mathrm{SNH}_{4} \mathrm{~N}_{5}\right)\left(\mathrm{CH}_{3} \mathrm{CV}\right)_{2},[2] \\
& \text { 2-Mercaptopyridine Carbon black (F) }
\end{aligned}
$$

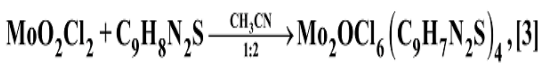

$$
\begin{aligned}
& \text { 4-Phenylimidazole-2-thiol Sand (R) } \\
& \mathrm{M}_{0} \mathrm{O}_{2} \mathrm{Cl}_{2}+\mathrm{C}_{5} \mathrm{H}_{4} \mathrm{~N}_{4} \mathrm{~S} . \mathrm{H}_{2} \mathrm{O} \underset{1: 2}{\stackrel{\mathrm{CH}_{3} \mathrm{CN}}{\longrightarrow}} \mathrm{M}_{2} \mathrm{O}_{2} \mathrm{Cl}_{8}\left(\mathrm{C}_{5} \mathrm{H}_{4} \mathrm{~N}_{4} \mathrm{~S}\right)\left(\mathrm{CH}_{3} \mathrm{CN}\right),[4] \\
& \text { 6-Mercaptopurine monohydrate Black (F) }
\end{aligned}
$$

\section{RESULTS AND DISCUSSIONS}

\section{Analytical Measurements

\begin{tabular}{|c|c|c|c|c|c|c|}
\hline Compounds & $\mathrm{Cl}$ & Mo & $\mathrm{H}$ & C & S & $\mathrm{N}$ \\
\hline $\begin{array}{l}\mathrm{MoOCl}_{3}\left(\mathrm{C}_{5} \mathrm{H}_{4} \mathrm{NS}-\mathrm{SNH}_{4} \mathrm{C}_{5}\right) \cdot 2 \mathrm{HCl},[1] \\
\quad(\text { Light blue green/511.5) }\end{array}$ & $\begin{array}{c}34.02 \\
(34.70)\end{array}$ & $\begin{array}{c}17.93 \\
(18.76)\end{array}$ & $\begin{array}{l}02.50 \\
(1.95)\end{array}$ & $\begin{array}{c}22.78 \\
(23.46)\end{array}$ & $\begin{array}{c}11.87 \\
(12.51)\end{array}$ & $\begin{array}{l}05.11 \\
(5.47)\end{array}$ \\
\hline $\begin{aligned} \mathrm{Mo}_{3} \mathrm{O}_{3} \mathrm{Cl}_{12}\left(\mathrm{C}_{5} \mathrm{H}_{4} \mathrm{NS}-\mathrm{SNH}_{4} \mathrm{C}_{5}\right)\left(\mathrm{CH}_{3} \mathrm{CN}\right)_{2}, \text { [2] } & (\text { Carbon black/1064.0) }\end{aligned}$ & $\begin{array}{c}39.78 \\
(40.03)\end{array}$ & $\begin{array}{c}27.59 \\
(27.06)\end{array}$ & $\begin{array}{l}01.95 \\
(1.31)\end{array}$ & $\begin{array}{c}15.58 \\
(15.78)\end{array}$ & $\begin{array}{l}05.67 \\
(6.01)\end{array}$ & $\begin{array}{l}04.77 \\
(5.26)\end{array}$ \\
\hline $\begin{aligned} \mathrm{Mo}_{2} \mathrm{OCl}_{6} & \left(\mathrm{C}_{9} \mathrm{H}_{7} \mathrm{~N}_{2} \mathrm{~S}\right)_{4},[3] \\
& (\mathrm{Sand} / 1121.0)\end{aligned}$ & $\begin{array}{c}19.78 \\
(19.00)\end{array}$ & $\begin{array}{c}16.28 \\
(17.12)\end{array}$ & $\begin{array}{l}02.88 \\
(2.49)\end{array}$ & $\begin{array}{c}38.97 \\
(38.53)\end{array}$ & $\begin{array}{c}12.32 \\
(11.41)\end{array}$ & $\begin{array}{l}10.08 \\
(9.99)\end{array}$ \\
\hline $\begin{array}{c}\mathrm{Mo}_{2} \mathrm{O}_{2} \mathrm{Cl}_{8}\left(\mathrm{C}_{5} \mathrm{H}_{4} \mathrm{~N}_{4} \mathrm{~S}\right)\left(\mathrm{CH}_{3} \mathrm{CN}\right),[4] \\
(\text { Black/701.0) }\end{array}$ & $\begin{array}{c}40.97 \\
(40.51)\end{array}$ & $\begin{array}{c}26.67 \\
(27.38)\end{array}$ & $\begin{array}{l}01.48 \\
(0.99)\end{array}$ & $\begin{array}{c}11.87 \\
(11.98)\end{array}$ & $\begin{array}{l}04.97 \\
(4.56)\end{array}$ & $\begin{array}{l}09.84 \\
(9.98)\end{array}$ \\
\hline
\end{tabular} \\ FTIR Spectra}

Table 1 shows the percentage of the elements. Theoretical values are enclosed in brackets.

Table 1: (Elemental Analysis)

\section{FTIR Spectra}

Table 2 shows that 2-mercaptopyridine ${ }^{35,44,48}$ has $\mathrm{N}-\mathrm{H}$ stretching $\left(3179 \mathrm{~cm}^{-1}\right)$ and $\mathrm{S}-\mathrm{H}$ stretching $\left(2709 \mathrm{~cm}^{-1}\right) .3208 \mathrm{~cm}^{-1}$ peak in [1] implies the presence of $\mathrm{N}-\mathrm{H}$ functional group. S-H peak at $\sim 2708 \mathrm{~cm}^{-1}$ disappears in [1] amounting to thiol group elimination. This further indicates that the ligand has reacted in [1] as thione. $\mathrm{C}=\mathrm{N}$ bond in [1] has absorbed at the same position as in the free ligand. There is positive shift of C-S frequency to $763 \mathrm{~cm}^{-1}$ due to increase in C-S bond order.
Absorption of terminal $\mathrm{Mo}=\mathrm{O}$ group $^{45-47}\left(983 \mathrm{~cm}^{-1}\right)$ is observed. $3209 \mathrm{~cm}^{-1}$ peak in [2] implies the presence of $\mathrm{N}-\mathrm{H}$ functional group. $\mathrm{S}-\mathrm{H}$ peak at $\sim 2709 \mathrm{~cm}^{-1}$ disappears in [2] amounting to thiol group elimination. This further indicates that the ligand has reacted in [2] as thione. $\mathrm{C}=\mathrm{N}$ bond in [2] has absorbed at $1599 \mathrm{~cm}^{-1}$ showing positive shift in frequency. There is positive shift of $\mathrm{C}$-S frequency to $760 \mathrm{~cm}^{-1}$ due to increase in $\mathrm{C}$-S bond order. Absorption of terminal $\mathrm{Mo}=\mathrm{O}$ group ${ }^{45-47}\left(974 \mathrm{~cm}^{-1}\right)$ is observed. 
Table 2: (Infrared frequencies in $\mathrm{cm}^{-1}$ )

\begin{tabular}{|c|c|c|c|}
\hline Mode & (2-Mercaptopyridine) $)^{35,44-48}$ & [1] & [2] \\
\hline$v(\mathrm{~N}-\mathrm{H})$ & 3179 & 3208.1 v s & 3209.4 v s \\
\hline$v(\mathrm{C}-\mathrm{H})$ & $3054,2929,2881$ & $3139.1 \mathrm{~s}, 3079.8 \mathrm{~s}, 2930.1 \mathrm{~m}$ & $3141.4 \mathrm{v} \mathrm{s}, 3087.4 \mathrm{~s}, 3023.4 \mathrm{~s}$ \\
\hline$v(S-H)$ & $2709 \mathrm{~m}$ & & \\
\hline$v_{s}(C-C$ ring $)$ & 1613 s, 1502 s, & & \\
\hline v (C-H Ring) & $1449 \mathrm{~s}, 1420 \mathrm{~s}$ & $1604.5 \mathrm{~s}, 1529.9 \mathrm{w}, 1509.1 \mathrm{~m}$ & $1607.3 \mathrm{~s}$ \\
\hline v $(\mathrm{C}=\mathrm{N})$ & $1585.6 \mathrm{~s}$ & $1585.6 \mathrm{~s}$ & $1599.3 \mathrm{~s}$ \\
\hline$v(\mathrm{C}-\mathrm{H}$ ring $)$ & $1248 \mathrm{~m}, 1188 \mathrm{vs}$ & $1220.2 \mathrm{w}$ & $1225.1 \mathrm{w}$ \\
\hline$v(\mathrm{C}-\mathrm{S})$ & 747 & 763.7 v s & 760.2 v s \\
\hline $\begin{array}{l}\text { Terminal } \\
v(\mathrm{Mo}=\mathrm{O})^{49-51}\end{array}$ & & $983.1 \mathrm{~s}$ & $974.4 \mathrm{~s}$ \\
\hline
\end{tabular}

$v_{s}=$ sym. stretching, $v=$ out-of-plane bending (wagging), $v=$ in-plane bending (rocking)

Table 3 shows $\mathrm{N}-\mathrm{H}$ stretching peak (3139 $\mathrm{cm}^{-1}$ ) in [3] of 4-phenylimidazole-2-thiol ${ }^{39,52-54}$. No S-H stretching in the range $2551-2603 \mathrm{~cm}^{-1}$ in [3] has been observed referring that $\mathrm{S}-\mathrm{H}$ group has got dissociated. Stretching of $\mathrm{C}=\mathrm{S}\left(1255 \mathrm{~cm}^{-1}\right.$ and $1225 \mathrm{~cm}^{-1}$ ) in [3] have been detected. Carbonyl group has greater stretching wave number than that of thio group due to the fact that $\mathrm{C}=\mathrm{O}$ group is stronger and more polar than $\mathrm{C}=\mathrm{S}$ group. Intensity of $\mathrm{C}=\mathrm{O}$ group absorptions is more than that of $\mathrm{C}=\mathrm{S}$ group. $\mathrm{C}-\mathrm{S}$ stretching $\left(761 \mathrm{~cm}^{-1}\right)$ has been manifested by [3] owing to origin of Mo-S bond. The latter has stretching at $529 \mathrm{~cm}-1 . \mathrm{Mo}=\mathrm{O}$ terminal group stretching $\left(992 \mathrm{~cm}^{-1}-1010 \mathrm{~cm}^{-1}\right)$ has been reported in literature. This peak was observed ${ }^{49-51}$ at 968 wave number in [3]. Imidazole-2-thiones ${ }^{39,54}$ undergoes thiol-thione tautomerism. There is decrease in $\mathrm{Mo}=\mathrm{O}$ stretching owing to $\mathrm{S} \rightarrow \mathrm{Mo}$ coordination $^{39,55} . \mathrm{Mo}=\mathrm{O}$ and ligand are trans to each other. It suggests that ligand reacts as thiol during the reaction. Higher wave number of $\mathrm{C}=\mathrm{N}$ stretching is associated with it.

Table 3: (Infrared frequencies in $\mathrm{cm}^{-1}$ )

\begin{tabular}{ccc}
\hline Mode & (4-Phenylimidazole-2-thiol) ${ }^{39,52-54}$ & [3] \\
\hline $\mathrm{N}-\mathrm{H}$ stretching & $3132,3247 \mathrm{~s}$ & $3139.3 \mathrm{~s}, 3050.2 \mathrm{~s}$ \\
$\mathrm{~S}-\mathrm{H}$ stretching & $1561,1503,1463$ & $1597.0 \mathrm{~s}, 1508.0 \mathrm{~m}, 1486.1 \mathrm{~m}, 1455.6 \mathrm{w}$ \\
$\mathrm{C}=\mathrm{N}, \mathrm{C}=\mathrm{C}$ stretching & 1261,1111 & $1255.0 \mathrm{~m}, 1225.2 \mathrm{w}, 1102.7$ \\
$\mathrm{C}=\mathrm{S}$ stretching & 783 & $761.7 \mathrm{v} \mathrm{s}$ \\
$\mathrm{C}-\mathrm{S}$ stretching & & $529.1 \mathrm{w}$ \\
Mo-S ${ }^{39,55}$ stretching & & $968.5 \mathrm{~s}$ \\
Terminal Mo=O & & \\
\hline
\end{tabular}

6-Mercaptopurine $e^{9,56,57}$ pyrimidine ring stretching $\left(3398 \mathrm{~cm}^{-1}\right)$ in [4] is indicative of $\mathrm{N}-\mathrm{H}$ group. 6-Mercaptopurine ${ }^{9,56,57}$ imidazole ring $\mathrm{N}-\mathrm{H}$ stretching is absent, because imidazole ring does not have $\mathrm{N}-\mathrm{H}$ bond in [4] (Table 4). This supports the presence of Mo-N bond. There is no S-H stretching in [4]. It is concluded that pyrimidine of 6-mercaptopurine reacted as thiol. This fact is also reflected by positive shift of $\mathrm{C}=\mathrm{N}$ stretching frequency and negative shift of C-S frequency. Here ligand may be acting as multidentate ligand as thione. $\mathrm{N} \rightarrow$ Mo coordination of pyrimidine decreases $\mathrm{N}-\mathrm{H}$ stretching. Mo-S stretching ${ }^{9,58}$ has been noticed at $730 \mathrm{~cm}^{-1}$ in [4]. Stretching at $977 \mathrm{~cm}^{-1}$ in [4] is concurrent with $\mathrm{Mo}=\mathrm{O}$ group in terminal position ${ }^{45-47}$.

\section{${ }^{1} \mathrm{H}$ NMR Spectra}

When 2-mercaptopyridine ${ }^{35,46}$ spectrum is compared with that of [1], it is observed that there is deshielding of protons due to $\mathrm{N} \rightarrow$ Mo coordination
(Table 5). $\mathrm{N}-\mathrm{H}$ resonance occurs at $14.93 \delta$. C-N bond $\pi$ electron density increases on coordination.

When 2-mercaptopyridine ${ }^{35,46}$ spectrum is compared with that of [2], it is observed that there is deshielding of protons due to $\mathrm{N} \rightarrow$ Mo coordination (Table 5). There is no $\mathrm{N}-\mathrm{H}$ peak. $\mathrm{C}-\mathrm{N}$ bond $\pi$ electron density increases on coordination. Notations $\downarrow \& \uparrow$ stand for shielding and deshielding.

Table 4 : (Infrared frequencies in $\mathrm{cm}^{-1}$ )

\begin{tabular}{ccc}
\hline Mode & $\begin{array}{c}\text { (6-Mercaptopurine } \\
\text { monohydrate) }\end{array}$ & {$[4] 56,57$} \\
\hline N-H Imidazole stretching & 3524 & \\
N-H Pyrimidine stretching & 3376 & $3398.5 \mathrm{v} \mathrm{s}$ \\
C-H stretching & $3095.0,2993.8$ & $3131.1 \mathrm{v} \mathrm{s}$ \\
S-H stretching & 2671.5 & \\
C=C stretching & 1669.7 & $1690.0 \mathrm{~s}$ \\
$\mathrm{C}=\mathrm{N}$ Imidazole stretching & 1620 & $1622.2 \mathrm{~s}$ \\
$\mathrm{C}=\mathrm{N}$ Pyrimidine stretching & 1393 & $1401.9 \mathrm{~m}$ \\
C-N stretching & 1343.8 & $1311.8 \mathrm{sh}$ \\
$\mathrm{N}-\mathrm{H}$ deformation & 1526.6 & $1520.3 \mathrm{sh}$ \\
C=S stretching & 1193 & $1027.9 \mathrm{~W}$ \\
Mo-S ${ }^{9,58}$ stretching & - & $730.0 \mathrm{~m}$ \\
Mo-N ${ }^{9}$ stretching & - & $562.2 \mathrm{~m}$ \\
Terminal Mo=O ${ }^{49-51}$ stretching & - & $977.0 \mathrm{~s}$ \\
\hline
\end{tabular}


Table 5: ('H NMR in $\delta)$

\begin{tabular}{lccc}
\hline Protons & (2-Mercaptopyridine) ${ }^{35,46}$ & {$[1]$} & [2] \\
\hline $\mathrm{N}-\mathrm{H}$ & & 14.93 & \\
$\mathrm{C} 3-\mathrm{H}$ & 7.31 & 7.67 & $7.61 \downarrow$ \\
$\mathrm{C} 4-\mathrm{H}$ & 6.82 & 7.28 & $7.23 \downarrow$ \\
$\mathrm{C} 5-\mathrm{H}$ & 7.48 & 8.04 & $7.75 \downarrow$ \\
$\mathrm{NC}-\mathrm{H}$ & 7.70 & 8.44 & $8.42 \downarrow$ \\
$\mathrm{CH}_{3} \mathrm{CN}$ & & & 1.86 \\
\hline
\end{tabular}

$\mathrm{N}-\mathrm{H}$ chemical shift of 4-phenylimidazole-2thio| ${ }^{39,59-60}$ appears at $12.9 \delta$. There is no particular chemical shift of alcoholic, amino, phenolic and thiolic protons in a solvent, because these protons are labile. There is no existence of $\mathrm{N}-\mathrm{H}$ and $\mathrm{S}-\mathrm{H}$ peaks in [3] due to above reasons. There is upfield chemical shift of ring protons and $\mathrm{H}-5$ due to $\mathrm{N} \rightarrow$ Mo coordination.

Table 6: ('H NMR in $\delta)$

\begin{tabular}{ccc}
\hline Protons & (4-Phenylimidazole-2-thiol) $^{39,59-60}$ & {$[3]$} \\
\hline $\mathrm{N}-\mathrm{H}$ & 12.98 & \\
$\mathrm{~S}-\mathrm{H}$ & 12.15 & \\
$\mathrm{H}-5$ & 7.52 & $7.39 \downarrow$ \\
Aromatic H-2 and H-6 & 8.14 & $7.67 \downarrow$ \\
Aromatic H-3 and H-5 & 7.52 & $7.30 \downarrow$ \\
Aromatic H-4 & 7.42 & $7.20 \downarrow$ \\
\hline
\end{tabular}

6-Mercaptopurine monohydrate ${ }^{57}$ aromatic $\mathrm{N}-\mathrm{H}$ absorption (Table 7) is not observed may be due to its participation in bonding. There is no $\mathrm{S}-\mathrm{H}$ peak may be due to reaction of the ligand as thione. Acetonitrile peak is observed at $2.05 \delta$.
Table 7: ('H NMR in $\delta)$

\begin{tabular}{lcc}
\hline Protons & (6-Mercaptopurine monohydrate) $^{57}$ & {$[4]$} \\
\hline $\mathrm{N}-\mathrm{H}$ (Arom) & 7.20 & \\
$\mathrm{~N}-\mathrm{H}$ & 1.41 & $1.76 \uparrow$ \\
$\mathrm{S}-\mathrm{H}$ & 1.22 & \\
$\mathrm{CH}_{3} \mathrm{CN}$ & & 2.05 \\
\hline
\end{tabular}

\section{${ }^{13} \mathrm{C}$ NMR Spectra}

In ${ }^{13} \mathrm{CNMR}$, the $\mathrm{C}=\mathrm{S}$ absorption of 2-mercaptopyridine ${ }^{46,61-63}$ in [2] is shifted upfield by 20 ppm (Table 8). This high chemical shift is characteristic of S-bonded thiones. This is due to $\mathrm{Mo} \rightarrow \mathrm{S}$ back donation of molybdenum d-orbitals to the sulfur empty $\pi^{\star}$-orbitals. It increases the $\mathrm{C}=\mathrm{S} \pi$-electron density and shielding of the thiocarbon. $\mathrm{C}-2$ has moved upfield while other carbons have moved downfield. Notations $\downarrow \& \uparrow$ stand for shielding and deshielding.

Table 8: $\left({ }^{13} \mathrm{C}\right.$ NMR in $\left.\delta\right)$

\begin{tabular}{ccc}
\hline Carbon & 2-mercaptopyridine ${ }^{46,61-63}$ & [2] \\
\hline C=S & 176.57 & $156.40 \downarrow$ \\
C-2 & 133.67 & $121.40 \downarrow$ \\
C-3 & 136.92 & $137.85 \downarrow$ \\
C-4 & 114.12 & $119.01 \downarrow$ \\
C-5 (attached to nitrogen) & 138.04 & $149.07 \downarrow$ \\
DMSO & 39.5 & 39.42 \\
\hline
\end{tabular}

\section{Mass Spectra (LC-MS) ${ }^{64}$}

Ionic species noted (Tables 9,10) justify the formulae.

Table 9: (LC-MS Ionization)

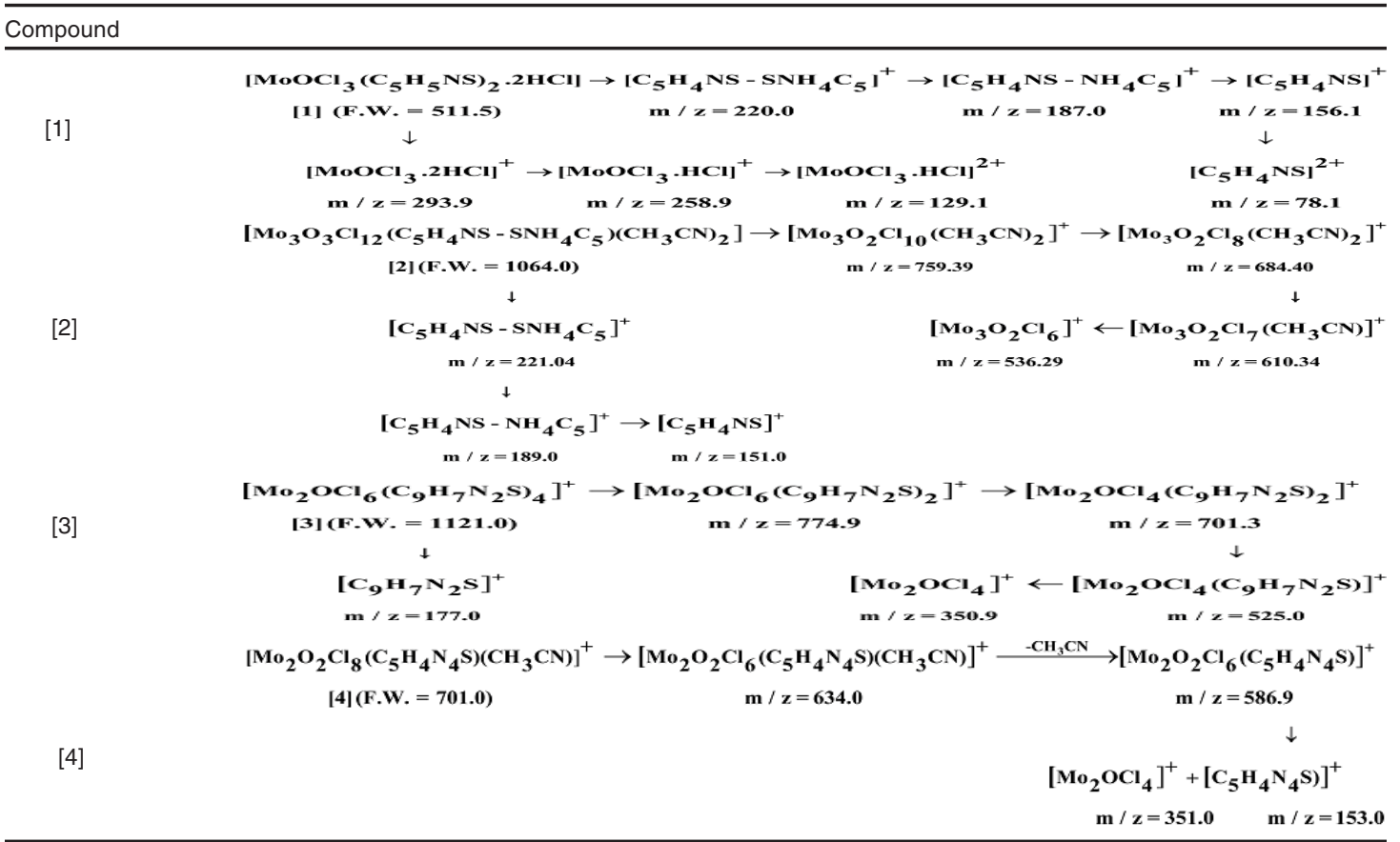


Table 10: (LC-MS Ion $\mathrm{m} / \mathrm{z}$ values)

\begin{tabular}{|c|c|c|c|c|}
\hline Compound & Ion & Calculated ${ }^{64}$ & Detected & Relative intensity \\
\hline \multirow[t]{7}{*}{ [1] } & {$\left[\mathrm{MoOCl}_{3} \cdot 2 \mathrm{HCl}\right]^{+}$} & 290.7 & 293.9 & $22 \%$ \\
\hline & {$\left[\mathrm{MoOCl}_{2} \cdot 2 \mathrm{HCl}\right]^{+}$} & 255.7 & 258.9 & $4 \%$ \\
\hline & {$\left[\mathrm{MoOCl}_{2} \cdot 2 \mathrm{HCl}\right]^{2+}$} & 127.8 & 129.1 & $6 \%$ \\
\hline & {$\left[\mathrm{C}_{5} \mathrm{H}_{4} \mathrm{NS}^{2} \mathrm{SNH}_{4} \mathrm{C}_{5}\right]^{+}$} & 220.1 & 220.0 & $100 \%$ \\
\hline & {$\left[\mathrm{C}_{5} \mathrm{H}_{4} \mathrm{NS}-\mathrm{NH}_{4} \mathrm{C}_{5}\right]^{+}$} & 188.0 & 187.0 & $22 \%$ \\
\hline & {$\left[\mathrm{C}_{5} \mathrm{H}_{4} \mathrm{~N}_{4} \mathrm{~S}\right]^{+}$} & 152.0 & 156.1 & $40 \%$ \\
\hline & {$\left[\mathrm{C}_{5} \mathrm{H}_{4} \mathrm{~N}_{4} \mathrm{~S}\right]^{2+}$} & 76.0 & 78.1 & $32 \%$ \\
\hline \multirow[t]{7}{*}{ [2] } & {$\left[\mathrm{C}_{5} \mathrm{H}_{4} \mathrm{~N}_{4} \mathrm{~S}\right]^{+}$} & 152.0 & 151.0 & $12 \%$ \\
\hline & {$\left[\mathrm{C}_{5} \mathrm{H}_{4} \mathrm{NS}-\mathrm{SNH}_{4} \mathrm{C}_{5}\right]^{+}$} & 220.1 & 221.0 & $100 \%$ \\
\hline & {$\left[\mathrm{C}_{5} \mathrm{H}_{4} \mathrm{NS}-\mathrm{NH}_{4} \mathrm{C}_{5}\right]^{+}$} & 188.0 & 189.0 & $32 \%$ \\
\hline & {$\left[\mathrm{Mo}_{3} \mathrm{O}_{2} \mathrm{Cl}_{10}\left(\mathrm{CH}_{3} \mathrm{CN}\right)_{2}\right]^{+}$} & 757.4 & 759.3 & $5 \%$ \\
\hline & {$\left[\mathrm{Mo}_{3} \mathrm{O}_{2} \mathrm{Cl}_{8}\left(\mathrm{CH}_{3} \mathrm{CN}\right)_{2}\right]^{+}$} & 687.5 & 684.4 & $7 \%$ \\
\hline & {$\left[\mathrm{Mo}_{3} \mathrm{O}_{2} \mathrm{Cl}_{7}\left(\mathrm{CH}_{3} \mathrm{CN}\right)\right]^{+}$} & 611.5 & 610.3 & $20 \%$ \\
\hline & {$\left[\mathrm{Mo}_{3} \mathrm{O}_{2} \mathrm{Cl}_{6}\right]^{+}$} & 535.1 & 536.2 & $17 \%$ \\
\hline \multirow[t]{5}{*}{ [3] } & {$\left[\mathrm{Mo}_{2} \mathrm{OCl}_{4}\right]^{+}$} & 351.6 & 350.9 & $100 \%$ \\
\hline & {$\left[\mathrm{C}_{9} \mathrm{H}_{8} \mathrm{~N}_{2} \mathrm{~S}\right]^{+}$} & 176.0 & 177.0 & $35 \%$ \\
\hline & {$\left[\mathrm{Mo}_{2} \mathrm{OCl}_{4}\left(\mathrm{C}_{9} \mathrm{H}_{7} \mathrm{~N}_{2} \mathrm{~S}\right)\right]^{+}$} & 526.7 & 525.0 & $5 \%$ \\
\hline & {$\left[\mathrm{Mo}_{2} \mathrm{OCl}_{6}\left(\mathrm{C}_{9} \mathrm{H}_{7} \mathrm{~N}_{2} \mathrm{~S}\right)_{2}\right]^{+}$} & 771.6 & 774.9 & $<1 \%$ \\
\hline & {$\left[\mathrm{Mo}_{2} \mathrm{OCl}_{4}\left(\mathrm{C}_{9} \mathrm{H}_{7} \mathrm{~N}_{2} \mathrm{~S}\right)_{2}\right]^{+}$} & 701.7 & 701.3 & $<1 \%$ \\
\hline \multirow[t]{5}{*}{ [4] } & {$\left[\mathrm{C}_{5} \mathrm{H}_{4} \mathrm{~N}_{4} \mathrm{~S}\right]^{+}$} & 152.0 & 153.0 & $100 \%$ \\
\hline & {$\left[\mathrm{Mo}_{2} \mathrm{O}_{2} \mathrm{Cl}_{6}\left(\mathrm{C}_{5} \mathrm{H}_{4} \mathrm{~N}_{4} \mathrm{~S}\right)\left(\mathrm{CH}_{3} \mathrm{CN}\right)\right]^{+}$} & 630.6 & 634.0 & $1 \%$ \\
\hline & {$\left[\mathrm{Mo}_{2} \mathrm{O}_{2} \mathrm{Cl}_{6}\left(\mathrm{C}_{5} \mathrm{H}_{4} \mathrm{~N}_{4} \mathrm{~S}\right)\right]^{+}$} & 589.6 & 586.9 & $1 \%$ \\
\hline & {$[\mathrm{Mo2OCl} 4]+$} & 351.6 & 351.0 & $46 \%$ \\
\hline & {$\left[\mathrm{Mo}_{2} \mathrm{O}_{2} \mathrm{Cl}_{8}\left(\mathrm{C}_{5} \mathrm{H}_{4} \mathrm{~N}_{4} \mathrm{~S}\right)\left(\mathrm{CH}_{3} \mathrm{CN}\right)\right]^{+}$} & 700.5 & 699.1 & $1 \%$ \\
\hline
\end{tabular}

\section{CONCLUSION}

$\mathrm{N}-\mathrm{H}$ group in [1] absorbs at $3208 \mathrm{~cm}^{-1}$. There is no peak in the region $2709 \mathrm{~cm}^{-1}$, because $\mathrm{S}-\mathrm{H}$ group has disappeared. There is positive shift of C-S frequency to $763 \mathrm{~cm}^{-1}$ due to increase in C-S bond order confirming the Mo-S bond formation. Ligand has reacted in [1] as thione. $\mathrm{Mo}=\mathrm{O}$ stretching at $983 \mathrm{~cm}^{-1}$ in [1] confirms that $\mathrm{Mo}=\mathrm{O}$ is situated at terminal position. Deshielding of protons shows that there is $\mathrm{N} \rightarrow$ Mo coordination. lons detected in GCmass spectrum convince the suggested formula.

[2] shows $\mathrm{N}-\mathrm{H}$ peak $\left(3209 \mathrm{~cm}^{-1}\right)$. There is no peak in the region $2709 \mathrm{~cm}^{-1}$, because S-H group has disappeared. There is positive shift of C-S frequency to $760 \mathrm{~cm}^{-1}$ due to increase in C-S bond order confirming the Mo-S bond formation. Ligand has reacted in [2] as thione. $\mathrm{Mo}=\mathrm{O}$ stretching at 974 $\mathrm{cm}^{-1}$ in [2] confirms that $\mathrm{Mo}=\mathrm{O}$ is situated at terminal position. Ions detected in LC-mass spectrum convince suggested formula. Deshielding of protons shows that there is $\mathrm{N} \rightarrow$ Mo coordination. Presence of $\mathrm{CH}_{3} \mathrm{CN}$ is confirmed by the presence of ${ }^{1} \mathrm{H}$ NMR peak at $1.86 \delta .{ }^{13} \mathrm{C}$ NMR also justifies the presence of 2-mercaptopyridine.

Thiol group is absent in [3] due to lack of absorptions in the frequency span 2549-2600.
Thiocarbonyl group $\left(1255,1225,1102 \mathrm{~cm}^{-1}\right)$ exists. C-S stretching $\left(761 \mathrm{~cm}^{-1}\right)$ manifests the origin of Mo-S bond. Mo=O terminal group (968 wave number) is noticed. $\mathrm{S}-\mathrm{H}$ chemical shift is missing due to absence of thiol group. Ions detected in LC-mass spectrum convince the suggested formula.

$\mathrm{S}-\mathrm{H}$ is lacking in [4] because of presence of $\mathrm{v}(\mathrm{C}=\mathrm{S})$ at $1027 \mathrm{~cm}^{-1}$ and absence of absorption $\sim 2672 \mathrm{~cm}^{-1}$. There is no S-H stretching in [4]. Pyrimidine of 6-mercaptopurine reacted as thiol. This fact is also reflected by positive shift of $\mathrm{C}=\mathrm{N}$ stretching frequency and negative shift of C-S frequency. Here ligand may be acting as multidentate ligand as thione. $\mathrm{N} \rightarrow$ Mo coordination of pyrimidine decreases $\mathrm{N}-\mathrm{H}$ stretching. There is $\mathrm{S} \rightarrow$ Mo coordination. $\mathrm{Mo}=\mathrm{O}$ terminal group $\left(977 \mathrm{~cm}^{-1}\right)$ is observed. There is no chemical shift of $\mathrm{S}-\mathrm{H}$ in ${ }^{1} \mathrm{H}$ NMR of [4]. Presence of $\mathrm{CH}_{3} \mathrm{CN}$ is confirmed by the presence of peak at $2.05 \delta$ in ${ }^{1} \mathrm{H}$ NMR. Derived formula is supported by LC-mass spectrum.

\section{ACKNOWLEDGEMENT}

We, the authors express our gratitude to P.U. Chandigarh, India for providing characterizing facility.

\section{Conflict of interest}

There is no conflict of interest among the authors. 


\section{REFERENCES}

1. https://medlineplus.gov/druginfo/meds/ a682653.html.

2. https://en.wikipedia.org/wiki/Mercaptopurine \#: : text=Mercaptopurine\%20(6\%2DMP)\% 2C,Crohn's\%20disease $\% 2$ C\%20and\%20 ulcerative $\% 20$ colitis.

3. Łakomska, I.; Pazderski, L.; Sitkowsk, J.; Kozerski, L.; Pełczynska, M.; Nasulewicz, A.; Opolski, A.; Szłyk, E., J. Mol. Struct., 2004, 707, 241-247.

4. Blank, C.; Dabrowiak, J., J. Inorg. Biochem., 1984, 21, 21-29.

5. Cuin, A.; Massabni, A. C.; Pereira, G. A.; Leite, C. Q. F.; Pavan, F. R.; Sesti-Costa, R.; Heinrich, T. A.; Costa-Neto, C. M., Biomed. Pharmacother., 2011, 65, 334-338.

6. Bariyanga, J.; Luyt, A., J. Mol. Struct., 2001, 559, 49-54.

7. Cini, R.; Cinquantini, A.; Sabat, M.; Marzilli, L. G., Inorg. Chem., 1985, 24, 3903-3908.

8. Dubler, E.; Gyr, E., Inorg. Chem., 1988, 27, 1466-1473.

9. Abeer A. Sharfalddin.; Emwas, A. H.; Jaremko, M.; Hussien, M. A., Appl Organomet Chem., 2021, 35, 1-18, DOI: 10.1002/aoc.6041.

10. Pazderski, L.; Łakomska, I.;Wojtczak, A.; Szłyk, E.; Sitkowski, J.; Kozerski, L.; Kamien'ski, B.; Koz'min'ski, W.; Tousek, J.; Marek, R., J. Molecular Structure., 2006, 785, 205-215.

11. Murai, T. Chemistry of Thioamides; Springer Nature Singapore Pte Ltd., 2019.

12. Owczarzak, A. M.; Kubicki, M., Acta Crystallographica, Section E., 2012, E68, 01686. doi:10.1107/S1600536812020090.

13. Al-R. K. A.; Abdel-Aziz, H. A., Molecules., 2010, 15, 3775-3815.

14. Borhani, D. W.; Calderwood, D. J.; Frank, K. E. H.; Davis, M.; Josephsohn, N. S.; Skinner, B. S. WO Pat. 2008/063287., 2008.

15. Fidanze, S. D.; Erickson, S. A.; Wang, G. T.; Mantei, R.; Clark, R. F.; Sorensen, B. K.; Bamaung, N. Y.; Kovar, P.; Johnson, E. F.; Swinger, K. K.; Stewart, K. D.; Zhang, Q.; Tucker, L. A.; Pappano, W. N.; Wilsbacher, J. L.; Wang, J., Sheppard, G. S.; Bell, R. L.; Davidsen, S. K.; Hubbard, R. D., Bioorg. Med. Chem. Lett., 2010, 20, 2452-2455.

16. Emmitte, K. A.; Wilson, B. J.; Baum, E. W.; Emerson, H. K.; Kuntz, K. W.; Nailor, K. E.;
Salovich, J. M.; Smith, S. C.; Cheung, M.; Gerding, R. M.; Stevens, K. L.; Uehling, D. E.; Jr. Mook, R. A.; Moorthy, G. S.; Dickerson, S. H.; Hassell, A. M.; Leesnitzer, M. A.; Shewchuk, L. M.; Groy, A.; Rowand, J. L.; Anderson, K.; Atkins, C. L.; Yang, J.; Sabbatini, P.; Kumar, R. Bioorg. Med. Chem. Lett., 2009, 19, 1004-1007.

17. Andreani, A.; Burnelli, S.; Granaiola, M.; Guardigli, M.; Leoni, A.; Locatelli, A.; Morigi, R.; Rambaldi, M.; Rizzoli, M.; Varoli, L.; Roda, A. Eur. J. Med. Chem., 2008, 43, 657-661.

18. Andreani, A.; Rambaldi, M.; Mascellani, G.; Rugarli, P., Eur. J. Med. Chem., 1987, 22, 19-22.

19. Amarouch, H.; Loiseau, P. R.; Bacha, C.; Caujolle, R.; Payard, M.; Loiseau, P. M.; Bories, C.; Gayral, P., Eur. J. Med. Chem., 1987, 22, 463-466.

20. Gupta, G. D.; Jain, K. K.; Gupta, R. P.; Pujari, H. K., Indian J. Chem., Sect. B: Org. Chem. Incl. Med. Chem., 1983, 22, 268.

21. Vu, C. B.; Bemis, J. E.; Disch, J. S.; Ng, P. Y.; Nunes, J. J.; Milne, J. C.; Carney, D. P.; Lynch, A. V.; Smith, J. J.; Lavu, S.; Lambert, P. D.; Gagne, D. J.; Jirousek, M. R.; Schenk, S.; Olefsky, J. M.; Perni, R. B., J. Med. Chem., 2009, 52, 1275-1283.

22. Andreani, A.; Rambaldi, M.; Andreani, F.; Bossa, R.; Galatulas, I., Eur. J. Med. Chem., 1988, 23, 385-389.

23. Andreani, A.; Rambaldi, M.; Locatelli, A.; Bossa, R.; Fraccari, A.; Galatulas, I., Pharm. Acta Helv., 1993, 68, 21-24.

24. Andreani, A.; Bonazzi, D.; Rambaldi, M., Arch. Pharm., 1982, 315, 451-456.

25. Andreani, A.; Rambaldi, M. M.; Locatelli, A.; Bossa, R.; Fraccari, A.; Galatulas, I., J. Med. Chem., 1992, 35, 4634-4637.

26. Ding, H.; Chen, Z.; Zhang, C.; Xin, T.; Wang, Y.; Song, H.; Jiang, Y.; Chen, Y. Xu, Y.; Tan, C. Molecules., 2012, 17, 4703-4716.

27. Abdelwareth Sarhan, A.O.; Al-Dhfyan, A.; Al-Mozaini, M. A.; Adra, C. N.; Aboul-Fadl, T. Eur. J. Med. Chem., 2010, 45, 2689-2694.

28. Poorrajab, F.; Ardestani, S. K.; Emami, S.; Behrouzi-Fardmoghaddam, M.; Shafiee, A.; Foroumadi, A. Eur. J. Med. Chem., 2009, 44, 1758-1762. 
29. Khalaj, A.; Nakhjiri, M.; Negahbani, A. S.; Samadizadeh, M.; Firoozpour, L.; Rajabalian, S.; Samadi, N.; Faramarzi, M. A.; Adipour, N.; Shafiee, A.; Foroumadi, A.; Eur. J. Med. Chem., 2011, 46, 65-70.

30. Kumaresan, K. L. Lu, S.; Wen, Y. S. ; Hwu, J. R., Organometallics, 1994, 13, 3170-3176.

31. Nagai, K.; Carter, B. J.; Xu, J.; Hecht, S. M., J. Am. Chem. Soc., 1991, 113, 5099-5100.

32. Lobana, T. S.; Bhatia, P. K., J. Sci. Ind. Res., 1989, 48, 394-401.

33. Singh, G.; Mangla, V.; Goyal, M.; Singla, K.; Rani, D., American International Journal of Research in Science, Technology, Engineering \& Mathematics., 2014, 8(2), 131-136.

34. Singh, G.; Mangla, V.; Goyal, M.; Singla, K.; Rani, D., American International Journal of Research in Science, Technology, Engineering \& Mathematics., 2015, 9(1), 25-33.

35. Singh, G.; Mangla, V.; Goyal, M.; Singla, K.; Rani, D., American International Journal of Research in Science, Technology, Engineering \& Mathematics., 2015, 10(4), 299-308.

36. Singh, G.; Mangla, V.; Goyal, M.; Singla, K.; Rani, D., International Congress on Chemical, Biological and Environmental Sciences., 2015, 930-942, 7-9, Kyoto (Japan).

37. Singh, G.; Mangla, V.; Goyal, M.; Singla, K.; Rani, D.; Kumar, R., American International Journal of Research in Science, Technology, Engineering \& Mathematics., 2016, 16(1), 56-64.

38. Singh, G.; Kumar, R., American International Journal of Research in Science, Technology, Engineering \& Mathematics., 2018, 22(1), 01-08.

39. Rani, D.; Singh, G.; Sharma, S., Orient. J. Chem., 2020, 36(6), 1096-1102.

40. Rani, D.; Singh, G.; Sharma, S., Orient. J. Chem., 2021, 37(1), 46-52.

41. Mangla, V.; Singh, G., American International Journal of Research in Science, Technology, Engineering \& Mathematics., 2019, 26(1), 145-148.

42. Mangla, V.; Singh, G., Orient. J. Chem., 2019, 35(3), 1094-1102.

43. Vogel, A. I., A Text Book of Quantitative Inorganic Analysis; John Wiley and Sons: New York, (Standard methods)., 1963.

44. http://www.sigmaaldrich.com/catalog/product/ aldrich/m5852?lang=en\&region=IN.

45. Refat, M. S.; Farias, R. F. D., J. Serb. Chem. Soc., 2006, 71(12), 1289-1300.
46. Hanif, M.; Saddiq, A.; Hasnain, S.; Ahmad, S.; Rabbani, G.; Isab, A. A., Spectroscopy., 2008, 22, 51-56.

47. Xhang, H. L.; Evans, S. D.; Henderson, J. R.; Miles, R. E.; Shen, T., J. Phys. Chem. B., 2003, 107, 6087-6095.

48. Shpakovsky, D. B.; Banti, C. N.; Houle, G. B.; Kourkoumelis, N.; Manoli, M.; Manos, M. J.; Tasiopoulos, A. J.; Hadjikakou, S. K.; Milaeva, E. R.; Charalabopoulos, K.; Bakas, T.; Butlerd, I. S.; Hadjiliadisa, N., Dalton Trans., 2012, 41, 14568-14582.

49. Barraclough, C. G.; Kew, D. J., Australian J. Chem., 1970, 23, 2387-2396.

50. Ward, B. G.; Stafford, F. E., Inorg. Chem., 1968, 7, 2569.

51. Bodo, H. H.; Regina, Z. Chem., 1976, 16, 407. 52. Jolley, J.; Cross, W. I.; Pritchard, R. G.; McAuliffe,C. A.; Nolan, K. B., Inorganica Chimica Acta., 2001, 315, 36-43.

53. Kahn, E. S.; Rheingold, A. L.; Shupack, S. I., J. Crystallographic and Spectroscopic Research., 1993, 23(9), 697-710.

54. Trzhtsinskaya, B. V.; Abramova, N. D., J. Sulphur Chemistry., 1991, 10(4), 389-430.

55. Abramenko, V. L.; Sergienko, V. S., Russian J. Inorg. Chem., 2009, 54(13), 2031-2053.

56. Kumar, G. P.; Sanganal, J. S.; Phani, A. R.; Tripathi, S. M.; Manohara, C.; Raghavendra, H. L.; Janardhana, P. B.; Amaresha, S.; Swamy, K. B.; Prasad, R. G. S. V., Phamacological Research., 2015, 100, 47-57.

57. http://www.molbase.com/en/hnmr_6857-347-moldata-838140.html\#tabs.

58. Ueyama, N.; Nakata, M.; Araki, T.; Nakamura, A., Chemical Soc. Japan, Chemistry Lett., 1979, 421-424.

59. Abramenko, V. L.; Sergienko, V. S.; Churakov, A. V., Russian J. Coord. Chem., 2000, 26(12), 866-871.

60. Sharma, M.; Koty, A.; Srivastava, M.; Srivastava, A., J. Chinese Chemical Society., 2007, 54, 1419-1432.

61. https://spectrabase.com/spectrum/ 1 wIN1BStPn8.

62. https://www.chemicalbook.com/Spectrum EN_2637-34-5_ ${ }^{13} \mathrm{CNMR} . \mathrm{htm}$.

63. https://pubchem.ncbi.nlm.nih.gov/compound/ 2-Mercaptopyridine\#section $={ }^{13} \mathrm{C}-\mathrm{NMR}$ Spectra.

64. http://www.sisweb.com/referenc/tools/ exactmass.htm. 